\title{
Chondrosarcoma of Posterior Nasal Septum
}

\author{
Gurung U, Joy L, Thapa NM, Pradhan B
}

Ganesh Man Singh Memorial Academy of ENT and Head \& Neck Studies

Tribhuwan University Teaching Hospital

Kathmandu, Nepal.

\section{Corresponding Author}

Urmila Gurung

Ganesh Man Singh Memorial Academy of ENT and Head \& Neck Studies

Tribhuwan University Teaching Hospital

Kathmandu, Nepal.

Email: dr.urmila.gurung@gmail.com

Citation

Gurung U, Joy L, Thapa NM, Pradhan B. Chondrosarcoma of Posterior Nasal Septum. Kathmandu Univ Med J 2012;38(2):89-91.

\begin{abstract}
Chondrosarcoma is a rare non-epithelial tumor comprising of $10-20 \%$ of primary bone tumor. The nasal septum is a rare site for its occurrence. We present one such case of chondrosarcoma of the nasal septum who was treated with endoscopic removal followed by post operative radiotherapy and discuss the relevant clinical presentation, diagnosis and treatment and review the literature also.
\end{abstract}

\section{KEYWORDS}

chondrosarcoma, excision, nasal septum

\section{INTRODUCTION}

Chondrosarcomas are non-epithelial malignant, slow growing, cartilaginous tumors accounting for $10 \%$ to $20 \%$ primary bone tumors of which only $5 \%$ to $10 \%$ occur in the head and neck region. ${ }^{1}$ The nasal septum as a site for chondrosarcoma is very rare with around only 50 cases reported in the literature..$^{1-3}$ The treatment of choice is surgical excision with use of adjuvant therapy under few circumstances..$^{1,2,4-7}$ Such a case of septal chondrosarcomas has not been reported yet in Nepal, hence, we present a case of nasal septal chondrosarcoma who was treated with endoscopic removal of the tumour followed by radiotherapy.

\section{CASE REPORT}

A 45 years old female presented to the ENT(Ear, Nose and Throat) opd with gradually progressive bilateral nasal obstruction of 6 months duration. Examination revealed a smooth pale mass seen at the posterior end of nasal cavity bilaterally. Rest of the ENT examination and ophthalmic examination were within normal limit. CT scan of the nose and paranasal sinuses showed midline destructive mass with specks of calcific foci. The mass occupied the nasal septum and both the nasal cavities and sphenoid sinus. Right lateral bony wall of sphenoid sinus was eroded and soft tissue involvement in the adjacent part was seen. Sella turcica was spared. Anterior extension of the mass involved ethmoidal sinus and downward extension involved floor of nasopharynx. Both maxillary sinuses were normal. The orbital wall and retrobulbar soft tissue attenuation was normal.(Fig 1) No cervical lymph node enlargement were noted. Endoscopic biopsy was taken which was suggestive of chondrosarcoma grade 1 . The patient then underwent endoscopic removal of the tumor. Per operatively, there was a firm to hard mass around $8 \times 6 \mathrm{~cm}$ in size arising from cartilaginous septum extending posteriorly and filling the entire nasopharynx and the sphenoid sinus, inferiorly extending upto the level of inferior turbinate. The mass was excised in piece meal along with posterior part of bony nasal septum. The sphenoid sinus was exposed.

Histopathological examination showed irregularly shaped 
lobules of chondroid tissue with mildly increased cellularity. Chondrocytes with hyperchromatic nuclei with prominent nucleoli in some binucleated cells were also seen with focal invasion into bone tissue and it was consistent with chondrosarcoma grade 1 (Fig 2). The patient then received external beam radiotherapy i.e 60G 30 fractions over six weeks period after consultation with a medical oncologist. Four months following her treatment she had no recurrence.(Fig 3)

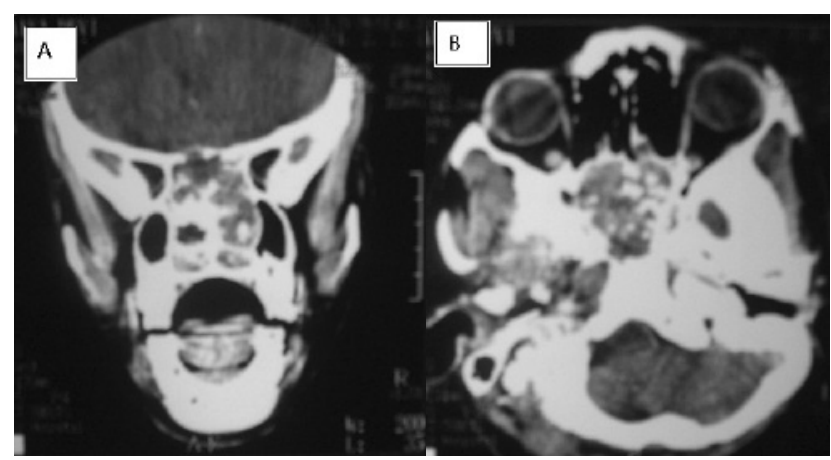

Figure 1. CT scan of PNS showing midline destructive mass with specks of calcific foci, occupying the nasal septum, both nasal cavities, ethmoid (A) and sphenoid sinus (B).

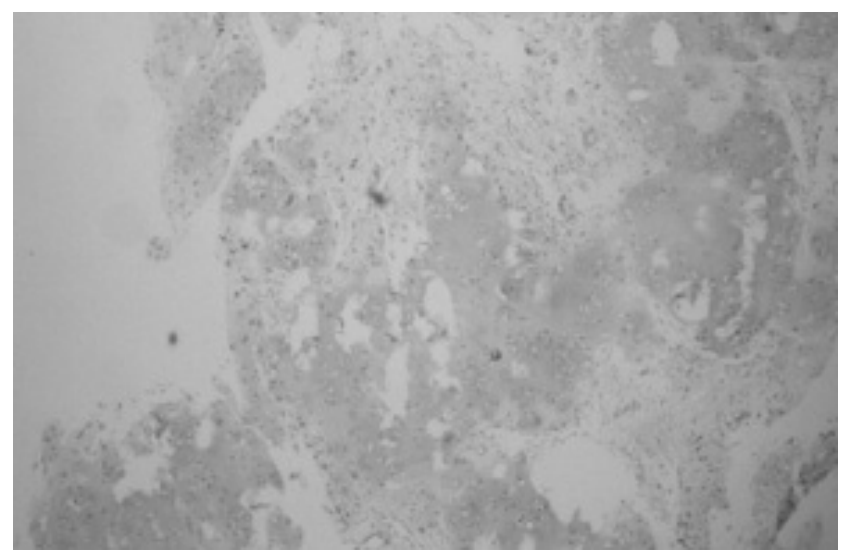

Figure 2. Chondrocytes with hyperchromatic nuclei with prominent nucleoli.

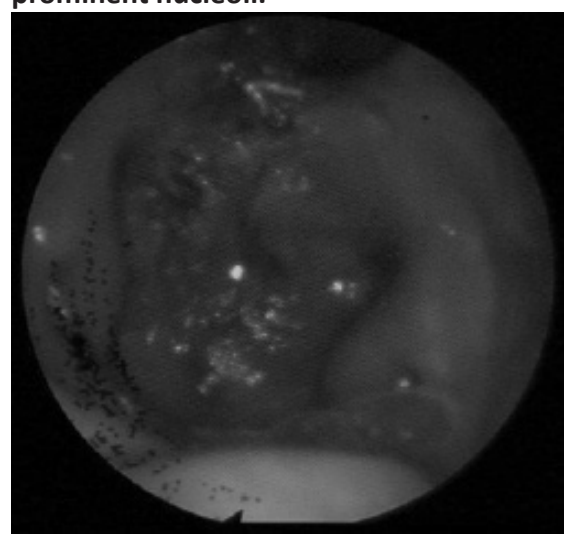

Figure 3. Endoscopic view of the nasopharynx showing normal bilateral eustachian tube openings (*) with absence of posterior end of nasal septum and no recurrence of tumour following endoscopic excision and post-operative radiotherapy.

\section{DISCUSSION}

Chondrosarcomas are rare tumors with the nasal septum being a rare site for its occurrence.

Chondrosarcomas are slow growing tumors so they often are large at the initial presentation. ${ }^{8}$ Symptoms depend on the site and size of the tumor, the common symptom being nasal obstruction. Others include epistaxis, anosmia, headache, facial pain, impaired vision, proptosis. ${ }^{1,8}$ Our patient presented with bilateral nasal obstruction only. The median age is fourth decade with male to female ratio varying from $1: 1$ to $10: 1$.

Diagnosis is made on the basis of histological examination. They can be graded on the basis of the degree of cellularity, nuclear size, atypia and mitotic activity. Grade 1 chondrosarcomas have abundant chondroid matrix, clusters of chondrocytes with normal or slightly enlarged nuclei, rare nucleoli, absent mitoses and occasional binucleation. Grade 2 chondrosarcomas appear to have less chondroid matrix and more chondrocytes than grade 1 tumors. It includes presence of rare mitosis, slightly enlarged vesicular hyperchromatic nuclei and multinucleation. Grade 3 chondrosarcoma display a myxoid matrix with irregularly shaped chondrocytes and increased cellularity, nuclear pleomorphism, and mitoses compared with low grade chondrosarcomas. ${ }^{1,2,4}$

Radiological imaging like CT scan and MRI are essential to evaluate the osseous and soft tissue characteristic of the tumor and for preoperative planning. ${ }^{3,4}$ On CT scan, chondrosarcomas classically appear as lesions composed of hypodense matrix with scattered small calcifications and associated bone erosion or destructions. ${ }^{2,3}$ The CT scan findings were similar in our patient. MRI, however helps to accurately define tumor extent and intracranial involvement and correlates well with the intraoperative findings. ${ }^{2}$ On MRI, they have a low intensity T1 imaging, high intensity $\mathrm{T} 2$ imaging and intermediate enhancement with T1 after gadolinium administration. , $3^{3}$

The clinical and radiological differential diagnosis includes chondroma, meningioma, osteomas, osteosarcomas, fibro-osseous lesions and chordoma. Histologically, chondrosarcomas need to be differentiated from chondroma, osteogenic sarcoma and salivary gland neoplasm. Chondromas may be difficult to be differentiated from chondrosarcomas. Hypercellularity and multinucleated cells with irregular hyperchromatic nuclei is useful to establish the diagnosis., ${ }^{3,6}$ Lesions greater than two $\mathrm{cm}$ are considered chondrosarcomas despite benign looking histology. ${ }^{6}$

Radical surgical resection is the definitive treatment of choice for chondrosarcomas. ${ }^{3,5}$ Various surgical approaches have been described in the literature like Weber Ferguson, sublabial-transnasal, lateral rhinotomy approach, Le Fort I downfracture and anterior cranial approach, transnasal endoscopic and microscopic approach. ${ }^{1,2,4,6}$ The use of 
navigation system with endoscopic surgery has also been tried.4 The endoscopic approach avoids facial and oral incisions producing less morbidity and thus better cosmetic result, maintains normal anatomic relationships and normal sinonasal functioning can be expected post operatively. ${ }^{2,6}$ However it has limitations due to the limited access provided by the nasal cavity and paranasal sinuses, the vascularity, size and extension of the tumor and availability of special instruments. ${ }^{6}$ Our patient underwent endoscopic removal of the tumour. Since, it was large, it had to be removed in piece meals.

Radiotherapy or chemotherapy are used for adjuvant treatment in case of residual disease, those extending to vital structure that impeded wide resection, recurrent disease and for palliation. ${ }^{1,6,7}$ Proton radiation therapy studies have shown excellent local control and good survival rate with skull base chondrosarcomas after surgical

\section{REFERENCES}

1. Downey TJ, Clark SK, Moore DW. Chondrosarcoma of the nasal septum. Otolaryngol Head Neck Surg 2001;125(1):98-100.

2. Coppit GL, Eusterman VD, Bartels J, Downey TJ. Endoscopic resection of chondrosarcomas of the nasal septum: a report of 2 cases. Otolaryngol Head Neck Surg 2002;127(6):569-71.

3. Yamamoto S, Motoori K, Takano H, Nagata H, Ueda T, Osaka I. Chondrosarcoma of the nasal septum. Skeletal Radiol 2002;31(9):5436.

4. Kainuma K, Netsu K, Asamura K et al. Chondrosarcoma of the nasal septum: A case report. Auris Nasus Larynx 2009;36(5):601-5.

5. Sakamoto T, Nakagawa T,Ito J. Endoscopic removal of chondrosarcoma originating from the nasal septum. The Open Otorhinolaryngology Journal [serial on the internet]. 2010 [cited 2010].4,1-4. Available from: http://www.benthamscience.com/open/tootorj/articles/ V004/1TOOTORJ.pdf debulking. ${ }^{1}$ In our case, CT scan had showed erosion of the right lateral bony wall of sphenoid sinus with soft tissue involvement in the adjacent part, hence the patient was sent for post operative radiotherapy.

Overall survival at five year for all grades combined is approximately 44 to $81 \% .^{1,3}$ At least $60 \%$ have a local recurrence within five years of initial treatment and the recurrence rate is as high as $65 \%$ with positive margin. ${ }^{1}$ Approximately $7-20 \%$ of the patients develop distant metastasis, mainly to lungs and bones..$^{1,3,4}$ Uncontrollable local disease is the most common cause of death. ${ }^{1,3}$ High grade, positive surgical margin, its location in the nasopharyx and posterior nasal cavity indicate poor prognosis. ${ }^{1}$

Chondrosarcomas of the nasal septum is a rare entity. Radiological investigations help in assessing its extent. Surgical excision is the treatment of choice with adjuvant therapy needed in selective cases only.

6. Carrau RL, Aydogan B, Hunt JL. Chondrosarcoma of the sphenoid sinus resected by an endoscopic approach. Am J Otolaryngol 2004;25(4):274-7.

7. Indudharan R, Das PK, Azman AA, Suhaiza S. Chondrosarcoma of the nasal septum. Singapore Med J 1998;39(8):376-9.

8. Aiyer RG, Prashanth V, Gupta R. Chondrosarcoma of the Nasal Septum. World articles in Ear, Nose and Throat [serial on the internet].2009 [cited on 2009 July 29] ;2(2). Available from: http:// www.waent.org/archives/2009/vol2-2/20090729-chrondrosarcoma/ chrondrosarcoma.htm 